Fiedler, Patrique; Griebel, Stefan; Biller, Sebastian; Fonseca, Carlos ; Vaz, Filipe;

Zentner, Lena; Zanow, Frank; Haueisen, Jens:

\title{
Novel cap system with active actuators for rapid dry electroencephalography
}

Zuerst erschienen in: $\quad$ Biomedical Engineering = Biomedizinische Technik. - Berlin [u.a.] : de Gruyter. - 58 (2013), Suppl. 1, Track-S, art. [4429], 2 pp.

Erstveröffentlichung: 2013-09-07

ISSN (online): $\quad$ 1862-278X

ISSN (print): $\quad$ 0013-5585

DOI: $\quad$ 10.1515/bmt-2013-4429

[Zuletzt gesehen: 2019-07-18]

„Im Rahmen der hochschulweiten Open-Access-Strategie für die Zweitveröffentlichung identifiziert durch die Universitätsbibliothek IImenau. “

"Within the academic Open Access Strategy identified for deposition by Ilmenau University Library."

„Dieser Beitrag ist mit Zustimmung des Rechteinhabers aufgrund einer (DFGgeförderten) Allianz- bzw. Nationallizenz frei zugänglich."

"This publication is with permission of the rights owner freely accessible due to an Alliance licence and a national licence (funded by the DFG, German

Research Foundation) respectively." 


\title{
Novel cap system with active actuators for rapid dry electroencephalography
}

\author{
Fiedler $\mathrm{P}^{1}$, Griebel $\mathrm{S}^{2}$, Biller $\mathrm{S}^{1}$, Fonseca $\mathrm{C}^{3}$, Vaz $\mathrm{F}^{4}$, Zentner $\mathrm{L}^{2}$, Zanow $\mathrm{F}^{5}$, Haueisen $\mathrm{J}^{1}$ \\ ${ }^{1}$ Institute of Biomedical Engineering and Informatics, Ilmenau University of Technology, Germany \\ ${ }^{2}$ Department of Mechanism Technology, Ilmenau University of Technology, Germany \\ ${ }^{3}$ Universidade do Porto, Faculdade de Engenharia, Rua Roberto Frias, 4200-465 Porto, Portugal \\ ${ }^{4}$ Universidade do Minho, Centro de Física, Campus de Azurém, 4800 Guimarães, Portugal \\ 5 eemagine Medical Imaging Solutions $\mathrm{GmbH}$, Berlin, Germany \\ patrique.fiedler@tu-ilmenau.de
}

\begin{abstract}
New fields of application for electroencephalography (EEG) require robust measurement technologies for ubiquitous mobile monitoring. Fast, easy and failsafe application as well as stable signal quality are crucial requirements for the electrodes. The application of novel dry EEG electrodes requires direct, reliable contact with the human skin as well as stable electrochemical characteristics of the materials. We propose a novel biocompatible electrode based on Titanium Nitride (TiN) integrated into an adaptive cap system with active adduction mechanisms. We report electrode-skin impedance measurements to prove our cap system to provide reliable and stable electrode positioning and adduction.
\end{abstract}

Keywords: Electroencephalography, Dry electrodes, Biosignal acquisition, Adaptive cap system, Active adduction mechanisms

\section{Introduction}

Silver/Silver-Chloride $(\mathrm{Ag} / \mathrm{AgCl})$ electrodes have been the gold standard in biosignal acquisition for several decades, but require extensive skin preparation and electrolyte gel application. Thus, they are inapplicable for upcoming new fields of application for electroencephalography (EEG) like Brain-Computer-Interfaces. Hence, dry electrode technologies have been developed for several years, eliminating the need for preparation and electrolyte gels or pastes [1]. Titanium-Nitride (TiN) based electrodes were shown to be mechanically and electrochemically stable in contact with human sweat [2]. EEG acquisition tests proved the signal quality of TiN electrodes to be comparable to conventional $\mathrm{Ag} / \mathrm{AgCl}$ electrodes [3].

The function principle of dry electrodes requires reliable, reproducible and stable adduction. In this paper we describe a novel overall cap concept based on TiN electrodes integrated into active actuators, ensuring the necessary homogenous and reproducible electrode adduction. Based on electrode-skin impedance measurements, we prove the function and effect of our cap system in a study on volunteers.

\section{Methods}

We developed a novel pin-shaped electrode whose top diameter of only $1.5 \mathrm{~mm}$ allows for a rapid, reproducible interfusion of the hair layer and a stable contact to the scalp (see Fig. 1a).
The substrate of $99.96 \%$ pure titanium (GoodFellow Metals, London, UK) was turned into final shape using a CNC lathe (PD CNC AD 1515, Spinner Werkzeugmaschinenfabrik $\mathrm{GmbH}$, Sauerlach, Germany). Subsequently a coating of a nano-sized layer of Titanium Nitride (TiN) was applied using a DC magnetron sputtering technique. Thus, the sensing element of the electrode exhibits excellent mechanical and electrochemical stability with good adhesion of the TiN coating [2]. The sensing element was connected to shielded coaxial cables using electrically conductive silver glue (Elecolit 325, Eurobond Adhesives Ltd, Sittingbourne, UK) and integrated into a silicone-based adduction actuator. The compliant actuator performs a translational movement if the internal pressure of the coherent, hollow structure is increased (see Fig. 1b). Hence, the adduction actuator allows for bypassing electrode-scalp distances of 2 to $8.5 \mathrm{~mm}$. a)

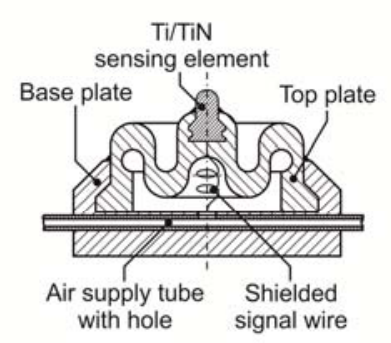

b)

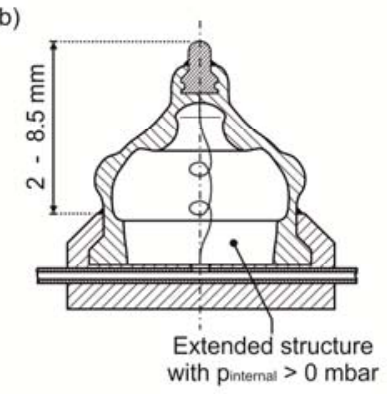

Figure 1: Novel pin-shaped TiN electrode integrated into an active adduction actuator: a) main components in initial position ( $p_{\text {internal }}=0$ mbar, without counter-pressure), and b) translational adduction of the actuator ( $p_{\text {internal }}>0$ mbar $)$.

Up to 165 electrode elements can be arranged and interconnected to form a high-density dry EEG cap system. In the present study, a total of 32 elements were used and arranged according to the international 10-20 system for EEG acquisition. All actuators were connected to a central pressure supply.

In order to evaluate the performance and reproducibility of the actuators and the electrode adduction, we performed electrode-skin impedance measurements on three volunteers with normal hair length and density at room temperature and $40 \%$ air humidity. Therefore, a commercial impedance ana- 
lyzer (4192A LF, Hewlett Packard, Palo Alto, USA) was used in combination with a custom-made security circuit. The impedance analyser is capable to measure impedances of $|\mathrm{Z}| \leq 1.3 \mathrm{M} \Omega$. The security circuit immediately cuts the connection of volunteer and measurement system if the measurement current eventually exceeds $50 \mu \mathrm{A}$.

Two conventional $\mathrm{Ag} / \mathrm{AgCl}$ electrodes were applied at $\mathrm{AFz}$ and $\mathrm{Fz}$ position on the head in combination with electrolyte gel (ECI Electro-Gel, Electro-Cap International Inc., Ohio, USA). The $\mathrm{Ag} / \mathrm{AgCl}$ to $\mathrm{Ag} / \mathrm{AgCl}$ impedance was always below $10 \mathrm{k} \Omega$. We sequentially measured the impedances between all dry $\mathrm{TiN}$ electrodes and the $\mathrm{Ag} / \mathrm{AgCl}$ electrode at $\mathrm{AFz}$ position. The measurement sequence was randomized in order to minimize the statistic influence of the time-tomeasurement on the impedance results. We repeated the measurement with increasing internal pressure applied to the actuator structures. For each set of parameters (including subject, pressure, electrode position) 65 samples were recorded at a sampling frequency of 2 samples / second. The 65 samples were subsequently averaged.

\section{Results}

Figure 2 shows the influence of the internal pressure of the actuators on the electrode-skin impedance. The number of active channels, i.e. $|\mathrm{Z}| \leq 1.3 \mathrm{M} \Omega$, continuously increases with increasing pressure. Due to the translational movement the sensing elements of the electrode better interfuse the hair layer. As the translation increases until electrode-skin contact is established, increasing pressure values allow for bridging of larger initial distances. As soon as the electrodes are in contact with the scalp, a further increase of the pressure causes decreasing impedance due to the electrode's tip causing an indentation in the scalp, thus leading to increased contact surface and better contact stability. The slightly increasing mean absolute impedance between 100 and 300 mbar is caused by the increased number of active channels rather than by increased per-electrode impedance.

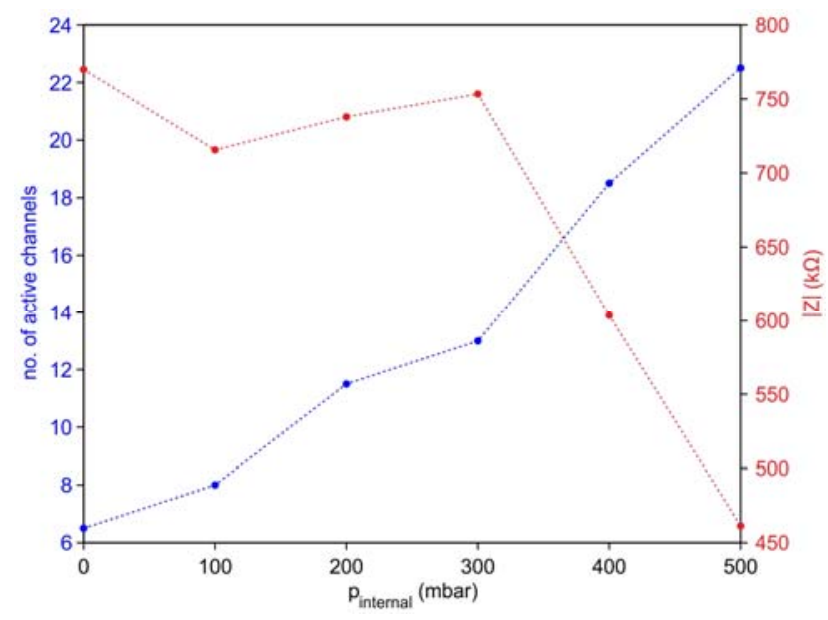

Figure 2: Influence of the applied internal pressure of the actuators on the electrode-skin impedance of three volunteers: number of active channels $(|Z|<1300 \mathrm{k} \Omega)$ shown in blue, and mean $|\mathrm{Z}|$ over all active channels shown in red.
Figure 3 shows a mapping of the measured electrode-skin impedances at different electrode positions. While the frontal electrodes exhibit electrode-skin contact even without adduction, the adduction effects with increasing pressure are clearly visible. The remaining non-contact and high-impedance electrode positions are caused by technological limitations of the current cap system. The electrodes at $\mathrm{F} 7$ and the auricular positions require a tighter cap design. The $\mathrm{O} 1$ and $\mathrm{P} 3$ electrodes are affected by drag forces of the weight of the bundled electrode cables. Finally, the parietal electrodes on the right side of the head may suffer from decreasing internal pressure due to the current pressure distribution network design.
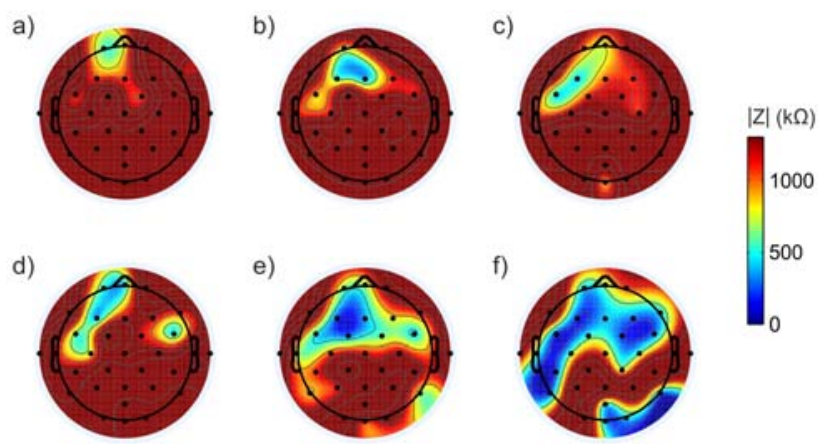

Figure 3: Mapping of the electrode-skin impedance (mean over all volunteers) for different internal pressure applied to the actuators: a) 0 mbar, b) 100 mbar, c) 200 mbar, d) 300 mbar, e) $400 \mathrm{mbar}$, and f) $500 \mathrm{mbar}$.

\section{Discussion}

We developed a novel cap system capable of enabling rapid dry EEG acquisition. Our experimental setup proved that the centrally controlled adduction actuators provide reproducible electrode-skin impedance. Furthermore, the translational movement and the applied contact pressure allow for increasing number of electrode-skin contacts and decreasing electrode-skin impedance. The biocompatible materials of the cap and the electrodes enable application in clinical routine as well as mobile and long-term measurements.

We will further improve the current cap design in order to eliminate current limitations like the lag of adduction at occipital and parietal electrode positions.

\section{Bibliography}

[1] Searle, A., and Kirkup, L.: A direct comparison of wet, dry and insulating bioelectric recording electrodes, Physiol. Meas., vol. 21, pp. 271-283, 2000

[2] Cunha, L.T., Pedrosa, P. et. al.: The role of composition, morphology and crystalline structure in the electrochemical behaviour of TiNx thin films for dry electrode sensor materials, Electrochim. Acta, vol. 55, pp. 59-67, 2009

[3] Fiedler, P., Cunha, L.T. et. al.: Novel TiNx-based biosignal electrodes for electroencephalography, Meas. Sci. Technol., vol. 22, article 025008, 2011 